MATEC Web of Conferences 11,02002 (2014)

DOI: $10.1051 /$ matecconf / 20141102002

(C) Owned by the authors, published by EDP Sciences, 2014

\title{
Analysis of elasto-plastic deformations of thick cylindrical tubes subject to pressures
}

\section{Analyse des déformations élasto-plastiques des tubes cylindriques épais soumis à des pressions}

\author{
A. ROUABHI \\ M’Hamed Bougara University, Départment of Civil Engineering, Boumerdes, Algeria
}

\begin{abstract}
This work consist in determining and analyzing analytically the stress and elasto-plastic strain state including the limit state using the theory of Huber-Mises-Henkey and formulating mathematically and solving the problem of determination of shearing lines their graphic representation. The analytical and graphical results show that the stress distribution for both limit plastic state and elastic state are different. At the limit state of plastic deformation the maximum stress perpendicular to the radius is reached at the external points of the tube, therefore it's possible to conclude that for the elasto-plastic materials the rupture starts from the outside, which is in good agreement with experimental results of the literature. The graphical representation of shearing lines has been obtained by means of a program developed, compiled and executed under Windows in the Visual Basic environment
\end{abstract}

\begin{abstract}
Résume: Ce travail consiste à déterminer et analyser analytiquement l'état de contraintes et de déformations élasto-plastiques y compris l'état limite en utilisant la théorie de Huber- Mises-Henkey et formuler mathématiquement et résoudre le problème de détermination des lignes de glissement avec leur représentation graphique. Les résultats analytiques et graphiques obtenus par cette théorie montrent que la répartition des contraintes à l'état plastique limite est différente de celle à l'état élastique. A l'état limite de déformation plastique la contrainte maximale perpendiculaire au rayon est atteinte aux points extérieurs du tube, et par suite il est possible de conclure que pour les matériaux élasto-plastiques la rupture commence à partir de l'extérieur, ce qui est conforme aux résultats expérimentaux de la littérature. La représentation graphique des lignes de glissement a été obtenue au moyen d'un programme élaboré, compilé et réalisé sous Windows dans l'environnement de Visual Basic.
\end{abstract}

\section{Introduction}

Les tubes cylindriques soumis à des pressions ont des domaines d'application très larges en génie civil et mécanique tels que les conduites d'eau, les gazoducs, les oléoducs, les turboréacteurs, les fusées, et même en biomécanique tels que les tubes minces et les vaisseaux sanguins. Le dimensionnement de ces corps reste toujours un axe de recherche important, Dans certaines situations, il est impossible de considérer toutes les contraintes qui agissent dans une structure comme appartenant au domaine élastique. Des contraintes supérieures à la limite d'élasticité du matériau peuvent se produire lorsqu'une structure est soumise à des sollicitations importantes. La structure doit alors résister sans s'effondrer, mais peut subir des déformations résiduelles permanentes [1]. Dans les secteurs industriels, les canalisations ont été employées en tant que moyens les plus économiques et les plus sûres pour transporter du pétrole et du gaz. Cependant, le nombre d'accidents a considérablement augmenté avec le nombre croissant de leur utilisation [2]. Donc Les canalisations sont des éléments de structures industrielles dont les dommages entrainent des risques pour les vies humaines et l'environnement écologique associé à des pertes d'exploitation avec souvent un préjudice financier qui peut être considérable [3].

L'examen des incidents sur tuyauteries ou tubes cylindriques montre que dans un certain nombre de situations, les méthodes usuelles de calcul de résistance ne permettent pas de faire un dimensionnement fiable. Ce travail consiste à considérer les déformations post élastiques, déterminer les zones élasto-plastiques correspondantes d'un tube cylindrique épais soumis à des chargements axisymétriques et analyser les contraintes 
limites, puis localiser les zones plastiques en fonction de l'épaisseur d'un corps cylindrique homogène soumis à une variation graduelle de pression. L'étude des déformations plastiques est basée sur le critère de plasticité de Huber-Mises-Hencky. En plus nous avons établi les équations différentielles des lignes de glissement ainsi que leur représentation graphique au moyen d'un programme élaboré en Visuel BASIC.

\section{Formulation mathématique du problème et sa solution analytique}

Considérons dans ce qui suit le problème de détermination des contraintes axi-symétriques dans un tube cylindrique infiniment long de rayon $\mathrm{r}$ compris entre le rayon interne $a$ et externe $b$ sous l'action d'une pression interne $\mathrm{Pb}$ à l'état élasto-plastique. Supposons que le matériau est élastique parfaitement plastique et incompressible. A l'état élastique, les contraintes normales sont déterminées par:

$$
\left\{\begin{array}{l}
\sigma_{r}=-\frac{p_{a} a^{2}}{b^{2}-a^{2}}\left(\frac{b^{2}}{r^{2}}-1\right) \\
\sigma_{\theta}=\frac{p_{a} a^{2}}{b^{2}-a^{2}}\left(\frac{b^{2}}{r^{2}}+1\right)
\end{array}\right.
$$

Dans le cas ou $\varepsilon_{z}$ est nulle, $\sigma_{z}=v\left(\sigma_{r}+\sigma_{\theta}\right)$ et comme le matériau est incompressible, alors la contrainte moyenne est nulle, ce qui implique que le coefficient de Poisson $v=0.5$.

A l'état elasto-plastique il est possible de considérer que la section du tube est composée de deux zones : une zone plastique où $\alpha \leq r \leq c$ et une autre élastique ou $c \leq r \leq b$.

En utilisant les formules (1). Nous pouvons écrire pour la zone élastique les expressions suivantes des contraintes normales [4], [5]:

$\left\{\begin{array}{l}\sigma_{r}=\frac{q c^{2}}{b^{2}-c^{2}}\left(\frac{b^{2}}{r^{2}}-1\right) \\ \sigma_{\theta}=\frac{q c^{2}}{b^{2}-c^{2}}\left(\frac{b^{2}}{r^{2}}+1\right)\end{array}\right.$

Où $q$ est la pression radiale à la frontière commune des zones élastique et plastique $(r=c)$. Pour la détermination des contraintes dans la zone plastique, nous utilisons en vertu de l'axisymétrie la seule équation d'équilibre suivante :

$\frac{d \sigma_{r}}{d r}+\frac{\sigma_{r}-\sigma_{\theta}}{r}=0$

qui doit être complétée par les conditions de plasticité. Les deux fameuses conditions de plasticité de HuberMises-Hencky et de Tresca-Saint-Venant donnent des résultats suffisamment proches, mais la condition de Huber-Mises-Hencky est mieux compatible avec les résultats expérimentaux. En plus la condition de HuberMises-Hencky est plus commode à utiliser du point de vue mathématique, puisque l'intensité des contraintes $\sigma_{i}$ s'exprime en fonction des composantes du tenseur des contraintes de façon relativement simple et par conséquent en théorie de plasticité on utilise le plus souvent la condition de Huber-Mises-Henkey [4],[6].

Dans notre cas l'intensité des contraintes aura la forme suivante :

$\sigma_{i}= \pm(\sqrt{3} / 2)\left(\sigma_{r}-\sigma_{\theta}\right)$

D'où puisque $\sigma_{\theta}$ est toujours supérieure à $\sigma_{\mathrm{r}}$ :

$\sigma_{\theta}-\sigma_{r}=2 \sigma_{T} / \sqrt{3}$

En résolvant simultanément les équations (3) et

(5) nous aurons :

$\frac{d \sigma_{r}}{d r}=\frac{2}{\sqrt{3}} \frac{\sigma_{T}}{r}$

L'intégration de cette équation différentielle à variables séparées permet d'avoir :

$\sigma_{r}=(2 / \sqrt{3}) \sigma_{T} \ln r+c$

En utilisant la condition au limite pour $r=a$,

$\sigma_{r}=-p$, nous obtenons l'expression de la constante :

$c=\left(-\frac{2}{\sqrt{3}}\right) \sigma_{T} \ln a-p$

En par suite su la base de (6) et (5) nous obtenons l'état des contraintes dans la zone plastique:

$\left\{\begin{array}{l}\sigma_{r}=-p+\frac{2}{\sqrt{3}} \sigma_{T} \ln \frac{r}{a} \\ \sigma_{\theta}=\sigma_{r}+\frac{2}{\sqrt{3}} \sigma_{T}\end{array}\right.$

La frontière entre la zone élastique et celle plastique les contraintes $\sigma_{r}$ et $\sigma_{\theta}$ doivent être continues. De l'égalisation des les parties droites des expressions correspondantes (2) et (7) pour $r=c$, nous obtenons deux équations pour la détermination de $q$ et $c$ :

$$
\begin{aligned}
& q=p-\frac{2}{\sqrt{3}} \sigma_{T} \ln \frac{c}{a} \\
& \ln \frac{c}{a}+\frac{1}{2}\left(1-\frac{c^{2}}{b^{2}}\right)=\frac{p \sqrt{3}}{2 \sigma_{T}}
\end{aligned}
$$

qui sert à déterminer le rayon $\mathrm{c}$ du cercle limitant la zone élastique et celle plastique. Après avoir déterminé $\mathrm{c}$ nous pouvons en utilisant la formule (8) déterminer la pression radiale $\mathrm{p}$.

Maintenant il est possible d'obtenir de façon générale les solutions des deux cas limites de déformation du tube :

\section{1 limite de déformation élastique}

Correspond à la valeur du rayon $\mathrm{c}=\alpha$. Alors de l'équation (9) nous obtenons la pression :

$P_{e l}=\frac{\sigma_{T}}{\sqrt{3}}\left(1-\frac{a^{2}}{b^{2}}\right)$

En portant cette valeur dans les formules (1) (solution élastique), nous obtenons les contraintes correspondantes:

$$
\left\{\begin{array}{l}
\sigma_{r}=\frac{\sigma_{T}}{\sqrt{3}} \frac{a^{2}}{b^{2}}\left(\frac{b^{2}}{r^{2}}-1\right) \\
\sigma_{\theta}=\frac{\sigma_{T}}{\sqrt{3}} \frac{a^{2}}{b^{2}}\left(\frac{b^{2}}{r^{2}}+1\right)
\end{array}\right.
$$


Les diagrammes des contraintes pour un tube de rapport $b / \alpha=2$ sont présentés à la figure 3 , dans ce cas :

$p_{e l}=0,433 \sigma_{T}=\frac{3}{4} \frac{\sigma_{T}}{\sqrt{3}}$

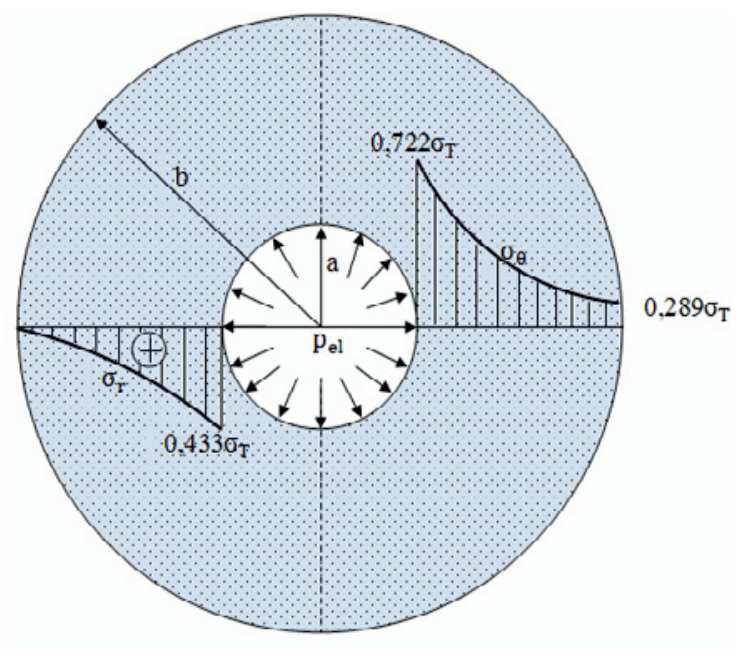

Fig.2

\subsection{Limite de déformation plastique :}

Ce cas correspond à $c=b$ ce qui donne :

$p_{T}=\frac{2}{\sqrt{3}} \sigma_{T} \ln \frac{b}{a}=0,800 \sigma_{T}$

Aprés avoir porté cette dernière expression dans la formule (7) nous obtenons les contraintes :

$\left\{\begin{array}{l}\sigma_{r}^{T}=-\frac{2}{\sqrt{3}} \sigma_{T} \ln \frac{b}{r} \\ \sigma_{\theta}^{T}=\frac{2}{\sqrt{3}} \sigma_{T}\left(1-\ln \frac{b}{r}\right)\end{array}\right.$

Les diagrammes des contraintes pour le même rapport $b / \alpha$ sont présentés à la figure 3 .

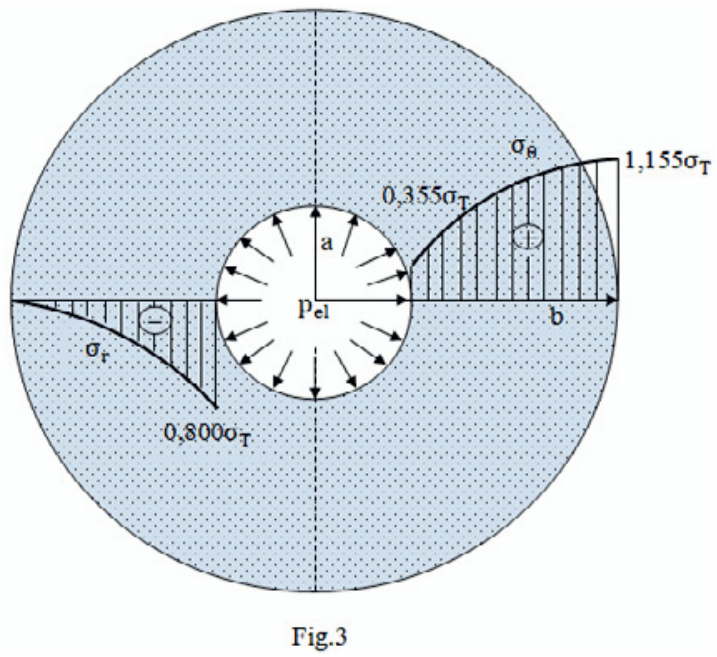

Dans ces cas la pression limite correspondante $P_{T}=0.800 \sigma_{T}$.
Si $p_{e l}<p<p_{T}$, alors le tube sera en état élasto-plastique. A la figure 4 sont présentés les diagrammes des contraintes $\sigma_{\theta}$ et $\sigma_{r}$ qui correspondent à la pression $P=0.700 \sigma_{T}$.

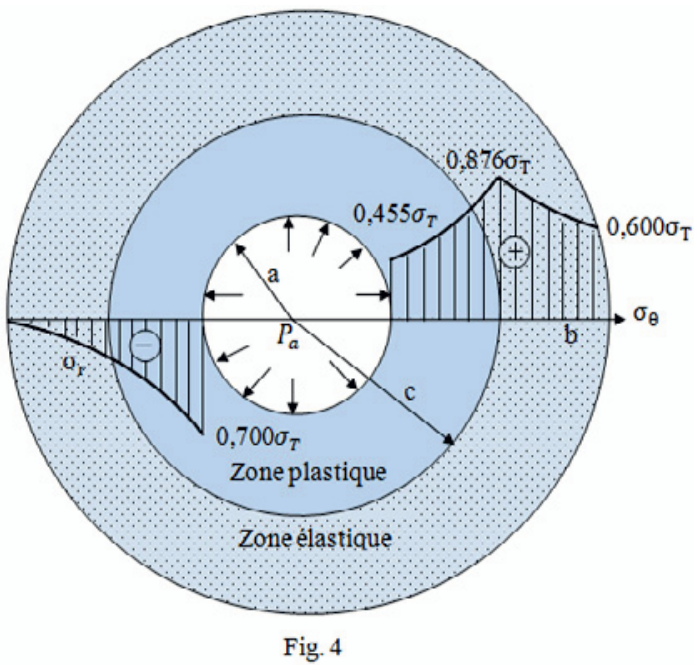

Dans ce cas le rayon de la frontière entre la zone élastique et plastique obtenue de l'équation (9) est $c=1.44 \mathrm{a}$. La pression radiale (8) a comme valeur $q=0.279 \sigma_{T}$. En portant la valeur de cette pression dans les formules (7), nous obtenons les expressions des contraintes dans la zone plastique du tube, (dans les limites $\alpha \leq r \leq 1.44 \alpha$ )

$\left\{\begin{array}{l}\sigma_{r}=\sigma_{T}\left(\begin{array}{c}\left.1,155 \ln \frac{r}{a}-0,700\right) \\ a\end{array}\right) ; p_{e l}<p<p_{T} \\ \sigma_{\theta}=\sigma_{r}+1,155 \sigma_{T}\end{array}\right.$

En appliquant la formule (2) pour les valeurs du rayon $c=1,44 a$, du rapport des rayons $\frac{b}{a}=2$ et de la pression $q=0,279 \sigma_{T}$, nous obtenons les expressions des contraintes dans la zone élastique du tube (dans les limites $1,44 a \leq r \leq 2 a$ ) :

$\left\{\begin{array}{l}\sigma_{r}=-0,300 \sigma_{T}\left(4 a^{2} / r^{2}-1\right) \\ \sigma_{\theta}=0,300 \sigma_{T}\left(4 a^{2} / r^{2}+1\right)\end{array}\right.$

Pour les points de la surface interne du tube (pour $r=a$ ), de la formule (13), nous obtenons :

$\left\{\begin{array}{l}\sigma_{r}=-0,700 \sigma_{T} \\ \sigma_{\theta}=0,455 \sigma_{T}\end{array}\right.$

Pour les points de la frontière entre la zone élastique et celle plastique $(r=1.44 \alpha)$, des formules (13) ou (14) nous aurons :

$\left\{\begin{array}{l}\sigma_{r}=-0,279 \sigma_{T} \\ \sigma_{\theta}=0,876 \sigma_{T}\end{array}\right.$

Pour les points de la surface externe du tube (pour $r=2 \alpha$ ), des formules (14) nous obtenons : 


$$
\left\{\begin{array}{l}
\sigma_{r}=0 \\
\sigma_{\theta}=0,600 \sigma_{T}
\end{array}\right.
$$

En analysant les diagrammes présentés sur les figures (2), (3) et (4), il est clair de voir que les contraintes normales radiales ne changent pas de caractère de distribution pendant le passage de l'état élastique à celle plastique, mais seulement leurs valeurs varient proportionnellement à la pression tandis que la répartition des contraintes normales perpendiculaires au rayon à l'état plastique change de façon brusque et elle est différente de celle à l'état élastique.

A l'état élastique les valeurs maximales des contraintes normales principales perpendiculaires aux rayons sont atteintes à la surface intérieure du tube tandis qu'a l'état limite plastique les valeurs de ces contraintes deviennent maximales à la surface extérieure du tube.

\section{Lignes de glissement}

Une attention particulière pour les problèmes de plasticité est réservée à la détermination des lignes de glissement ou de cisaillement Ces dernières sont des lignes dont la ligne tangente en chaque point détermine la direction de la contrainte tangentielle maximale.

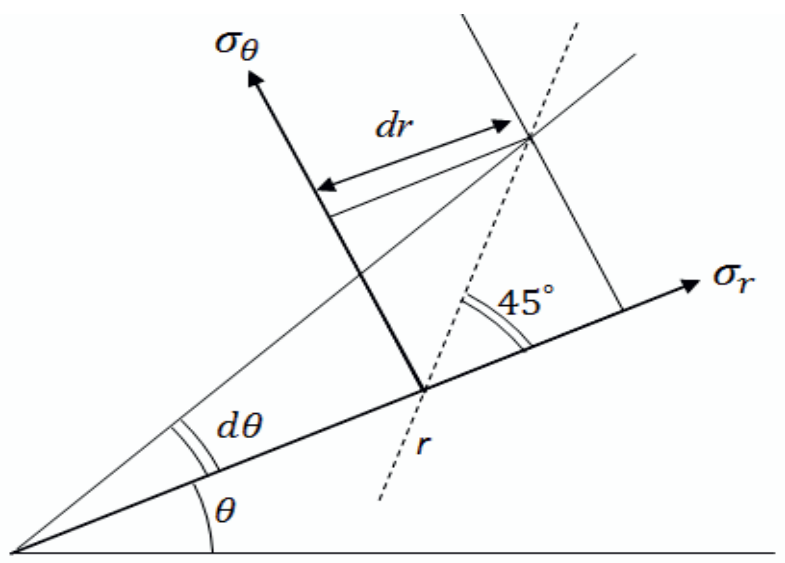

Fig. 5

Pour un tube cylindrique long, les contraintes principales dans une section transversales sont dirigées selon la direction radiale et celle perpendiculaire à cette dernière (les contraintes tangentielles selon ces directions sont nulles), et par conséquent les lignes de glissement dans une section droite se trouvant à l'état plastique sont inclinés à 45 degrés par rapport à ces directions et forment deux ensembles de lignes orthogonales (voir figure 5), [4], [6].Le long du tube ces lignes forment les surfaces de glissement.

Il faut noter que les conditions de plasticité de Huber-Mises et celles de Saint-venant pour l'état plan de déformation sont identiques à une constante près et par conséquent les théories de l'état plan de déformation plastique basées sur ces conditions se confondent.
La géométrie de la figure 5 permet d'écrire : $d r= \pm r d \theta$. Cette dernière est équation différentielle ordinaire dont la solution représente deux ensembles de lignes orthogonales de glissement de fonction en coordonnées polaires $r=c^{e \pm \theta}$ qui représentent géométriquement des spirales logarithmiques. La représentation graphique de cette solution est obtenue a l'aide d'un programme préparé en Visuel Basic. Les tracés des lignes de glissement montrés ci-dessous en sont des exemples.

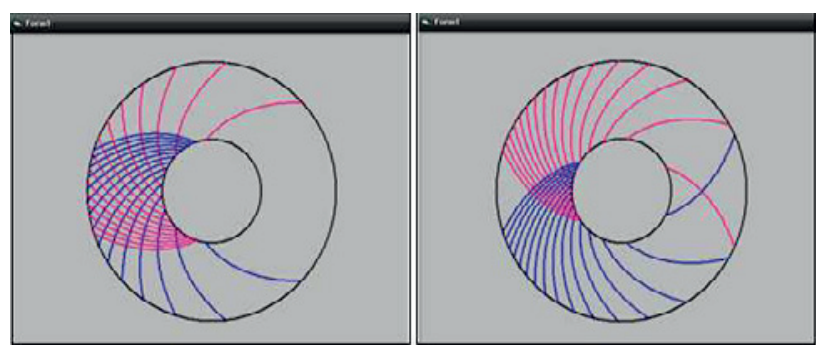

Orthogonalité des lignes de glissement pour $\theta>0$ e t $\theta<0$

Dans le cas d'un état plan de déformation les axes principaux forment avec l'axe $x$ les angles $\theta$ et $\theta+\pi / 2$ et les tangentes aux lignes de glissement perpendiculaires en tout point sont inclinées par rapport à l'axe $x$ d'un angle $\alpha^{*}$ et $\alpha^{*}+\pi / 2$ respectivement ,ce qui permet d'écrire : $\theta=\alpha^{*}-\pi / 2$.

Il est possible de montrer que dans le cas de déformation plane, tout élément limité par des lignes de glissement est soumis à une traction uniforme $\sigma_{0}$ selon ces lignes.

\section{Conclusion}

Selon les résultats présentés, il est claire de voir qu'à l'état élastique, les couches internes du tube sont les plus sollicitées par contre à l'état plastique ce sont les couches externes qui se trouvent dans des conditions critiques, ce qui permet de conclure que la rupture commence à partir de l'extérieur. L'augmentation de l'épaisseur des tubes ne peut pas assurer la résistance requise à de hautes pressions. Une des solutions constructives permettant d'augmenter la capacité portante est l'utilisation des tubes composés et composites.

\section{Références}

1. R .Hill, The Mathematical Theory of Plasticity, (1950). 2. A..El-hakimi, S. Hariri, M. Adj-meliani, Z. Azari, Etude sous pression de tuyaux de transport de gaz en présence de defauts de type entaille. Laboratoire de Fiabilité Mécanique (LFM), Université de Metz- ENIM, Ile du Saulcy, 57045 Metz. (Août 2007).

3. T. Boukharouba, Etude du comportement en fatigue des fissures semi-elliptiques, application aux plaques en flexion et aux tubes sous pression interne ».Thèse Doctorat, Université de Metz (04 juillet 1995).

4. L.Katchanov. Eléments de la théorie de plasticité, Editions mir-Moscou (1975)

5. G. Duvant, Mécanique du Milieu continu, Masson, Paris (1990). 